\title{
Pleural/Elastic Layer Invasion
}

National Cancer Institute

\section{Source}

National Cancer Institute. Pleural/Elastic Layer Invasion. NCI Thesaurus. Code C106305.

A pathologic finding indicating the extent of visceral pleural invasion by a malignant neoplasm. 